\title{
Nanoporous Nickel Microspheres: Synthesis and Application for the Electrocatalytic Oxidation and Determination of Acyclovir
}

\author{
Hossein Heli, ${ }^{* 1 \dagger}$ Fatemeh Pourbahman, ${ }^{* 2}$ and Naghmeh SattarahmadY $* 3, * 4$ \\ *1 Laboratory of Analytical and Physical Electrochemistry, Department of Chemistry, Science and Research \\ Branch, Islamic Azad University, Fars, 7348113111, Iran \\ *2 Department of Chemistry, Islamic Azad University, Firouzabad Branch, Firouzabad, Iran \\ *3 Department of Medical Physics, School of Medicine, Shiraz University of Medical Sciences, Shiraz, Iran \\ *4 Pharmaceutical Science Research Center, Shiraz University of Medical Sciences, Shiraz, Iran
}

\begin{abstract}
Nickel microspheres were synthesized via a water-in-oil reverse nanoemulsion system using nickel nitrate as the nickel precursor and hydrazine hydrate as the reducing agent. The nanoemulsion was a triton X-100/cyclohexane/water ternary system. The surface morphology of the nickel microspheres was studied by scanning electron microscopy, which indicated that the microspheres had a nanoporous structure. The electrochemical behavior of the nanoporous nickel microspheres were studied in alkaline solution and were then employed to fabricate a modified carbon paste electrode in order to investigate the electrocatalytic oxidation of the drug acyclovir. The oxidation process involved, and its kinetics were investigated using cyclic voltammetry and chronoamperometry. The rate constant of the catalytic oxidation of acyclovir and the electron-transfer coefficient are reported. A sensitive, simple and time-saving amperometric procedure was developed for the analysis of acyclovir. The proposed amperometric method was also applied to determine acyclovir in tablets and topical cream.
\end{abstract}

(Received December 12, 2011; Accepted March 9, 2012; Published May 10, 2012)

\section{Introduction}

Metal and metal oxide nanostructures are currently attracting considerable research interest in many fields, such as biomedical, optical, electrochemical and electronics. ${ }^{1-6}$ This is due to the high surface area, and also unique size-dependent properties of these materials, such as quantum confinement, plasmon resonance, and superparamagnetism. Specifically, the nanostructured materials can represent excellent electronic conductivity as well as catalytic and electrocatalytic properties, which cause acceleration of electron transfer between the electrode surface and the redox species. In this regards, a variety of nanostructured nickel-based materials with different morphologies, such as nanowires, nanorods, nanotubes, nanowhiskers, and nanoparticles, have been successfully synthesized..$^{7-12}$ In particular, the design and fabrication of nanoporous (including microporous, mesoporous and macroporous materials) nickel-based materials have attracted considerable attention in recent years..$^{13-17}$

Micro- and nanoemulsions as new micro-reactors have been widely used in the production of metallic nanoparticles, semiconductor, metal oxides and nanometer-scale magnetic particles. ${ }^{18-21}$ Water-in-oil microemulsions comprise oil and aqueous phases as well as a surfactant in which the polar group of the surfactant is concentrated in the interior, and the lipophilic group extends outwards into the nonpolar solvent. These reverse

† To whom correspondence should be addressed.

E-mail: hheli7@yahoo.com micelles are heterogeneous on a molecular scale and are thermodynamically stable. The chemical reaction in these micro- and nanoemulsions occurs at the oil/water interface, and hence the microemulsions are suitable reaction media for the synthesis of nanostructured materials. The aqueous phase inside these reverse micelles acts as a micro-reactor for performing synthesis reactions, in which the size and shape of the products are controlled by the size of the individual micelle. ${ }^{18-21}$ In addition, the better size distributions of synthesized materials can also be achieved in this media.

Carbon paste electrodes (CPEs) are used greatly in electrochemical studies due to their advantages, such as easy preparation, modification with different compounds, renewability, low cost, wide potential window, and low-charging current. Modified CPEs can be prepared using various compounds, such as transition metals and the corresponding oxides, metal complexes and biological compounds. ${ }^{22}$ The metal particle-modified CPEs represent both advantages of carbon paste electrodes and electrocatalytic reactivity of metals.

\section{Experimental}

\section{Reagents and chemicals}

All other chemicals used in this work (otherwise those stated) were of analytical grade from Merck, and were used without further purification. Carbon micro-particles (graphite fine powder) with a particle size of less than $50 \mu \mathrm{m}$ were obtained from Merck. Nafion solution was purchased from Sigma. All of the solutions were prepared by redistilled water. Acyclovir 
was received as a gift from Arasto Pharmaceutical Chemicals Inc., Tehran, Iran. The acyclovir tablets/topical creams were purchased from a local drugstore.

\section{Apparatus}

Electrochemical measurements were carried out in a conventional three-electrode cell containing a $100 \mathrm{mmol} \mathrm{L}^{-1}$ $\mathrm{NaOH}$ solution (which was employed as the supporting electrolyte throughout the work) powered by a Potentiostat/Galvanostat, an Autolab PGSTAT 302N (EcoChemie, Netherlands). $\mathrm{Ag} / \mathrm{AgCl} / 3 \mathrm{~mol} \mathrm{~L}^{-1} \mathrm{KCl}$ and a platinum plate were used as the reference and counter electrodes, respectively. The system was operated by a PC through GPES software.

Scanning electron microscopy (SEM) was performed using a X-30 Philips Instrument. X-ray diffraction (XRD) patterns were measured using a Philips X'Pert, the Netherlands, through $\mathrm{Cu} \mathrm{K}_{\alpha}$ radiation at $40 \mathrm{kV}$ and $30 \mathrm{~mA}$.

\section{Synthesis of nanoporous nickel microspheres}

Nanoporous nickel microspheres were synthesized from a Triton X-100/cyclohexane/water reverse nanoemulsion system. ${ }^{20}$ The processes of synthesis and sample manipulations were carried out in an inert atmosphere (nitrogen). Two nanoemulsion media were prepared, named I and II. Nanoemulsion I was composed of $2 \mathrm{mmol}$ Triton $\mathrm{X}-100+25 \mathrm{~mL}$ cyclohexane $+1.0 \mathrm{mmol} \mathrm{Ni}\left(\mathrm{NO}_{3}\right)_{2} \cdot 2 \mathrm{H}_{2} \mathrm{O}$ dissolved in $5 \mathrm{~mL}$ of water. Nanoemulsion II was composed of 2 mmol Triton X-100 + $25 \mathrm{~mL}$ cyclohexane $+3.0 \mathrm{mmol} \mathrm{NaOH}$ dissolved in $5 \mathrm{~mL}$ of water + $10 \mathrm{~mL}$ hydrazine hydrate. Two nanoemulsions were added together, and the obtained mixture was stirred for $2 \mathrm{~h}$, while entering nitrogen gas into the mixture. A solid black product was precipitated after that time. The product was collected and washed with redistilled water and ethanol, respectively.

\section{Preparation of the working electrodes}

A bare carbon paste electrode (CPE) was prepared by hand-mixing carbon microparticles and mineral oil with an $80 / 20 \%(w / w)$ ratio. The paste was carefully mixed and homogenized in an agate mortar for $20 \mathrm{~min}$. The resulting paste was kept at room temperature in a desiccator before use. The paste was packed firmly into a cavity $(4.05 \mathrm{~mm}$ diameter, geometric surface are of $0.128 \mathrm{~cm}^{2}$ and $0.5 \mathrm{~mm} \mathrm{depth}$ ) at the end of a Teflon tube. Electrical contact was established via a copper wire connected to the paste in the inner hole of the tube. The electrode surface was gently smoothed by rubbing on a piece of weighing paper just prior to use. This procedure was also used to regenerate the surface of CPE.

A nanoporous nickel microspheres-modified carbon paste electrode (MCPE) was prepared by hand-mixing carbon microparticles, mineral oil and nanoporous nickel microspheres with a 70/20/10\% (w/w) ratio. The modified electrode was then transferred to a $100 \mathrm{mmol} \mathrm{L}^{-1} \mathrm{NaOH}$ solution and treated by applying 25 consecutive cyclic potentials in a regime of cyclic voltammetry in a range of -100 to $700 \mathrm{mV}$ at a potential sweep rate of $50 \mathrm{mV} \mathrm{s}^{-1}$. This procedure leads to some structural changes and transformation of the nickel to the corresponding nickel oxide.

A nanoporous nickel microspheres-modified carbon paste electrode covered by Nafion (MCPE/Nf) was prepared by covering MCPE with $10 \mu \mathrm{L}$ of a $2 \%$ (w/v) low aliphatic alcohols Nafion solution.

\section{Procedures}

Standard solutions of the drug were prepared by dissolving an accurate mass of the bulk drug in an appropriate volume of $100 \mathrm{mmol} \mathrm{L}^{-1} \mathrm{NaOH}$ solution (which was also used as the supporting electrolyte). All of the solutions were kept in the dark at $4^{\circ} \mathrm{C}$, and used within $24 \mathrm{~h}$ to avoid decomposition. Additional dilute solutions were prepared daily by accurate dilution just before use.

The calibration curve for the drug in a $100 \mathrm{mmol} \mathrm{L}^{-1} \mathrm{NaOH}$ solution was measured with an amperometric technique. The working potential of $550 \mathrm{mV}$ was used in amperometric measurements. All the studies/measurements were carried out at room temperature.

\section{Acyclovir tablet and topical cream assay procedures}

For analysis of the tablets, the average weight of ten tablets from each sample was determined; then, the tablets were finely powdered and homogenized in a mortar. Appropriate and accurately weighed amounts of the homogenized powder were transferred into $25 \mathrm{~mL}$ calibrated flasks containing $15 \mathrm{~mL}$ of a $100 \mathrm{mmol} \mathrm{L}^{-1} \mathrm{NaOH}$ solution. The contents of the flasks were stirred for $24 \mathrm{~h}$, and then the undissolved excipients were removed by filtration and diluted to volume with the same supporting electrolyte. Appropriate solutions were prepared by taking suitable aliquots of the clear filtrate, and diluting them with a $100 \mathrm{mmol} \mathrm{L}^{-1} \mathrm{NaOH}$ solution.

For analysis of the topical cream, a suitable amount of cream was transferred into a beaker, and then $10 \mathrm{~mL}$ of a $100 \mathrm{mmol} \mathrm{L}^{-1}$ $\mathrm{NaOH}$ solution was added. The contents of the beaker were stirred for $24 \mathrm{~h}$, and then the undissolved excipients were removed by filtration. Appropriate solutions were prepared by taking suitable aliquots of the clear filtrate and diluting them with a $100 \mathrm{mmol} \mathrm{L}^{-1} \mathrm{NaOH}$ solution.

\section{Results and Discussion}

Electron microscopy

Figure 1 shows a SEM image of nanoporous nickel microspheres. Nickel microspheres with an average size of about $500 \mathrm{~nm}$ can be observed over the entire area, and the microspheres contain nanopores on the surface.

\section{XRD pattern}

Figure 2 shows an XRD pattern of the synthesized nanoporous nickel microspheres. The main diffraction peaks at about $45^{\circ}$, $52^{\circ}$ and $76^{\circ}$ are indexed to $\left(\begin{array}{lll}1 & 1 & 1\end{array}\right),\left(\begin{array}{lll}2 & 0 & 0\end{array}\right)$ and (2 200$)$ reflections, respectively. The diffraction peaks in the pattern can be indexed to a pure fcc crystal structure of nickel (Fm3m, JCPDS 87-0172). No other impurities can be detected from this pattern. This indicates that well-crystallized nickel nanowires can be easily obtained under the current synthetic condition.

\section{Study of the kinetics of charge transfer across the MCPE/solution} interface

When a nanoporous nickel microspheres-modified carbon paste electrode was transferred to the supporting electrolyte and treated by applying a potential in a regime of consecutive cyclic voltammetry, electrochemical oxidation of nickel to $\mathrm{Ni}(\mathrm{II})$ species was performed:

$$
\mathrm{Ni}+2 \mathrm{OH}^{-} \longrightarrow \mathrm{Ni}(\mathrm{OH})_{2}+2 \mathrm{e}^{-}
$$

This reaction is irreversible, and the nickel particles were transformed to the hydroxide one after several potential sweeps.

Figure 3A shows cyclic voltammograms of MCPE in a $100 \mathrm{mmol} \mathrm{L}^{-1} \mathrm{NaOH}$ solution recorded at a wide range of 


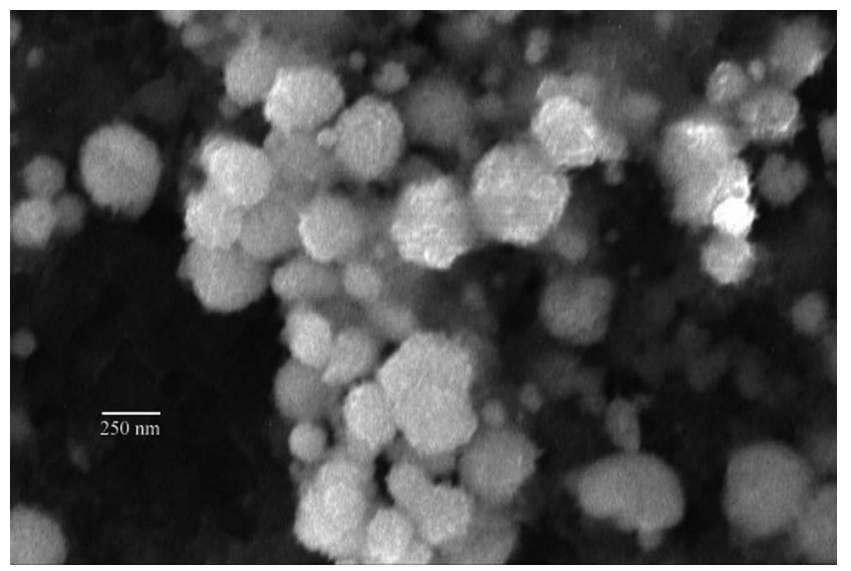

Fig. 1 A SEM image of nanoporous nickel microspheres.

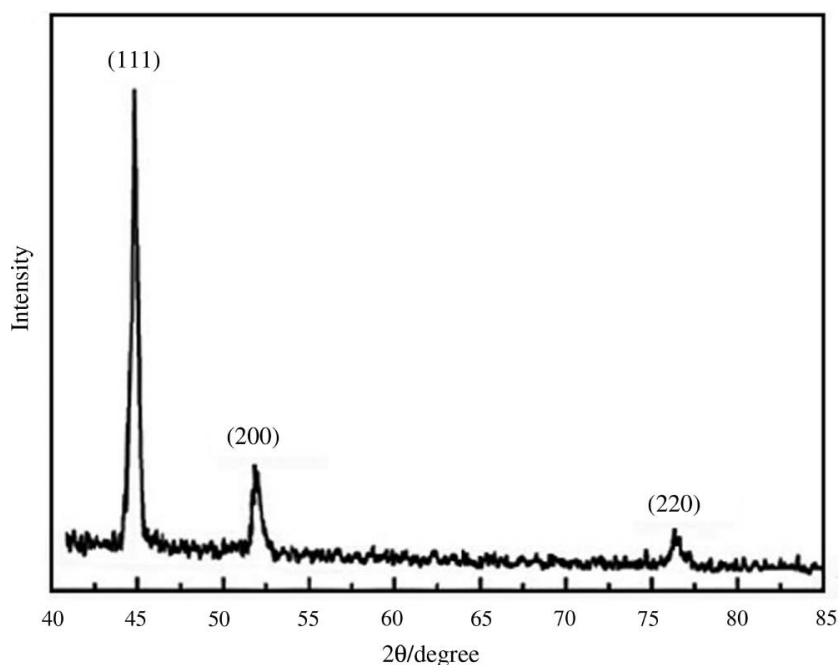

Fig. 2 An XRD pattern of the as-synthesized nanoporous nickel microspheres
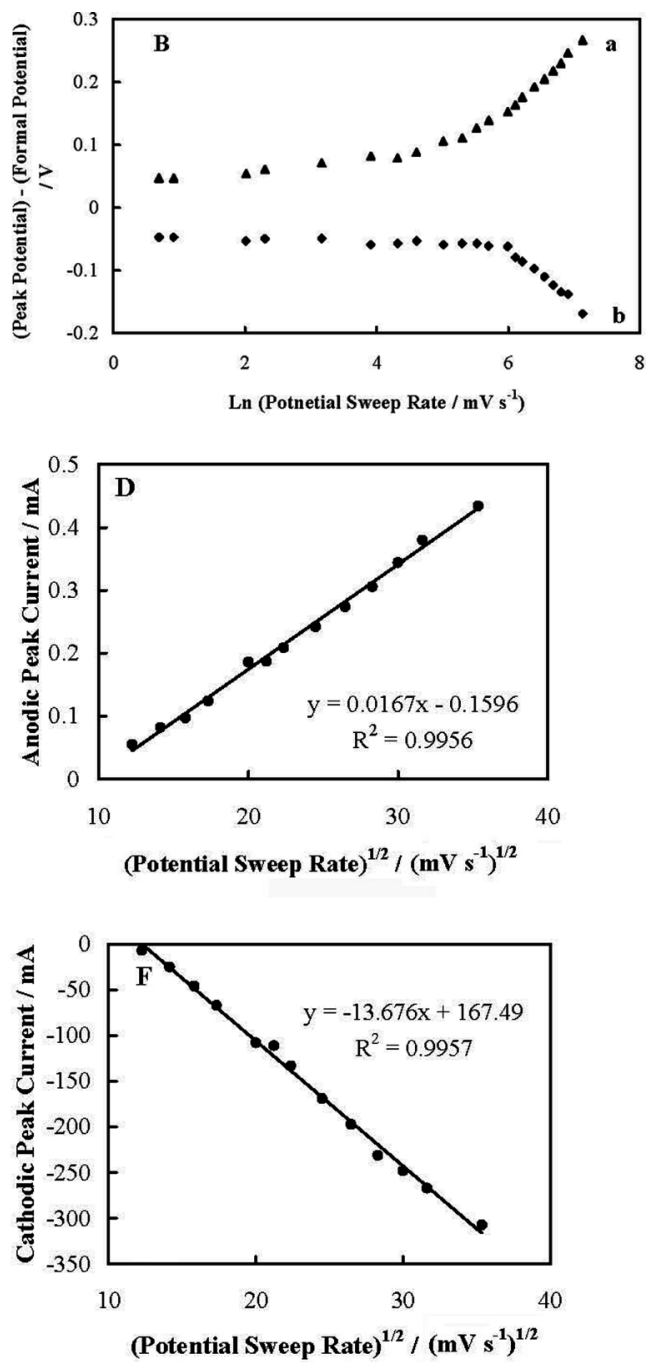

Fig. 3 (A) Cyclic voltammograms of MCPE in $100 \mathrm{mmol} \mathrm{L}^{-1} \mathrm{NaOH}$ solution recorded at different potential sweep rates. The potential sweep rates from the inner to the outer were: $2,2.5,5,7.5,10,20,50$, $75,100,150,200,250,300,350,400,450,500,600,700,800,900,1000$ and $1250 \mathrm{mV} \mathrm{s}^{-1}$. (B) Plot of $\left(E_{\mathrm{p}}-E^{0^{\prime}}\right)$ with respect to the logarithm of the potential sweep rate for the anodic (a) and cathodic (b) peaks. (C-F) Dependency of anodic (C) and cathodic (E) peak currents of the cyclic voltammograms shown in (A) on the potential sweep rate at low values of 2 to $100 \mathrm{mV} \mathrm{s}^{-1}$ and the dependency of anodic (D) and cathodic (F) peak currents on the square root of the potential sweep rate at high values of 150 to $1250 \mathrm{mV} \mathrm{s}^{-1}$. 
potential sweep rates, from 2 to $1250 \mathrm{mV} \mathrm{s}^{-1}$. A pair of well-defined peaks with a mid-peak potential of $444 \pm 11 \mathrm{mV}$ appeared in the voltammograms, and the peak-to-peak potential separation (at the potential sweep rate of $10 \mathrm{mV} \mathrm{s}^{-1}$ ) was $145 \mathrm{mV}$. The voltammograms shown are similar to those previously reported, ${ }^{10,23-25}$ and the redox transition involved is attributed to the presence of the $\mathrm{Ni}(\mathrm{II}) / \mathrm{Ni}(\mathrm{III})$ species immobilized at the electrode surface via the following reaction: ${ }^{10,23-25}$

$$
\mathrm{NiOOH}+\mathrm{H}_{2} \mathrm{O}+\mathrm{e}^{-} \rightleftarrows \mathrm{Ni}(\mathrm{OH})_{2}+\mathrm{OH}^{-}
$$

The peak-to-peak potential separation in the voltammograms deviated from the theoretical value of zero, and increased at higher potential sweep rates. This result indicates a limitation in the charge-transfer kinetics, which is due to: (a) chemical interactions between the electrolyte ions and the nickel species, (b) the dominance of electrostatic factors, (c) lateral interactions of the redox couples present on the surface, and/or (d) non-equivalent sites present in the nickel species.

Laviron derived general expressions for the linear potential sweep voltammetric response of surface-confined electro-reactive species at small concentrations. ${ }^{26}$ The expressions for the peak-to-peak potential separation $\left(\Delta E_{\mathrm{p}}\right)>200 / n \mathrm{mV}$, where $n$ is the number of exchanged electrons, are as follows:

$$
\begin{aligned}
& E_{\mathrm{pa}}=E^{0^{\prime}}+X \ln \left[\left(1-\alpha_{\mathrm{s}}\right) / m\right] \\
& E_{\mathrm{pc}}=E^{0^{\prime}}+Y \ln \left[\alpha_{\mathrm{s}} / m\right] \\
& \ln k_{\mathrm{s}}=\alpha_{\mathrm{s}} \ln \left(1-\alpha_{\mathrm{s}}\right)+\left(1-\alpha_{\mathrm{s}}\right) \ln \alpha_{\mathrm{s}}-\ln (R T / n F v)- \\
& \quad \alpha_{\mathrm{s}}\left(1-\alpha_{\mathrm{s}}\right) n F \Delta E_{\mathrm{p}} / R T
\end{aligned}
$$

where $X=R T /\left(1-\alpha_{\mathrm{s}}\right) n F, Y=R T / \alpha_{\mathrm{s}} n F, m=(R T / F)\left(k_{\mathrm{s}} / n v\right), E_{\mathrm{pa}}$ and $E_{\mathrm{pc}}$ are the anodic and cathodic peak potentials, respectively. $\alpha_{\mathrm{s}}, k_{\mathrm{s}}$ and $v$ are the electron-transfer coefficient, apparent charge-transfer rate constant and potential sweep rate, respectively. From these expressions, $\alpha_{\mathrm{s}}$ can be determined by measuring the variation of the peak potential with respect to the potential sweep rate, and $k_{\mathrm{s}}$ can be determined for electron transfer between the electrode and the surface-deposited layer by measuring the $\Delta E_{\mathrm{p}}$ values. Figure $3 \mathrm{~B}$ shows a plot of $\left(E_{\mathrm{p}}-E^{0^{\prime}}\right)$ with respect to the logarithm $v$ from the cyclic voltammograms represented in Fig. 3A for the anodic (curve a) and cathodic (curve b) peaks. It can be observed that for potential sweep rates of 300 to $750 \mathrm{mV} \mathrm{s}^{-1}$, the values of $\left(E_{\mathrm{p}}-E^{0^{\prime}}\right)$ linearly depend on the logarithm of the potential sweep rate, indicated by Laviron. Using the plot and Eqs. (2) to (4), the values of $\alpha_{\mathrm{s}}$ for the anodic and cathode peaks were obtained as $0.72 \pm 0.03$ and $0.28 \pm 0.03$, respectively. This discrepancy suggests that the rate-limiting steps for the reduction and oxidation processes might not be the same. ${ }^{27}$ Moreover, the mean value of $k_{\mathrm{s}}$ was obtained as $3.48 \pm 0.06 \mathrm{~s}^{-1}$. This is higher than those reported elsewhere in the literature ${ }^{28,29}$ for the redox process of nickel species generated from nickel bulk metal. This result indicates that the nanoporous structure represents a higher electron-transfer rate in the redox process of nickel species in an alkaline solution compared to the microstructures represented in Refs. 28 and 29. The voltammograms represented in Fig. 3A also show that the anodic and cathodic peak currents depend linearly on the potential sweep rate at low values of 2 to $100 \mathrm{mV} \mathrm{s}^{-1}$ (Figs. 3C and 3E). This result is attributable to the electrochemical activity of an immobilized redox couple at the surface. From the slope of these lines, and using ${ }^{30}$
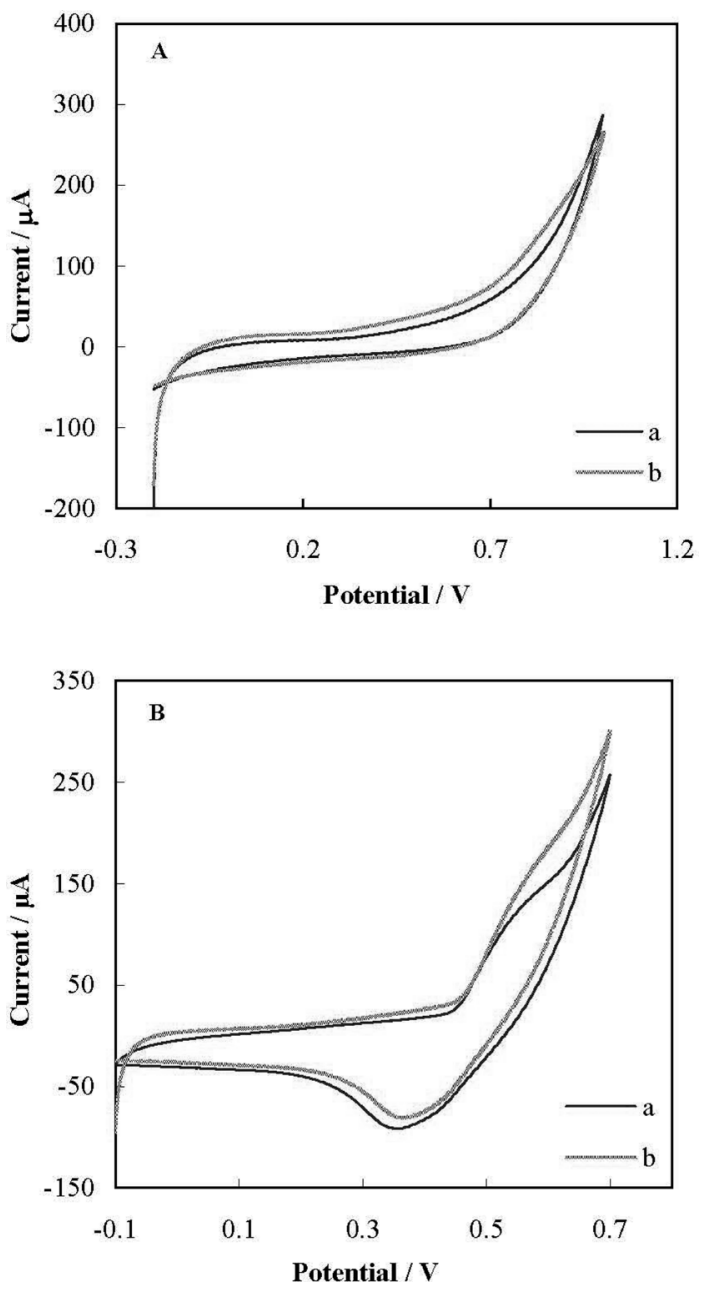

Fig. 4 (A) Typical cyclic voltammograms of CPE recorded in the absence (a) and presence (b) of $300 \mu \mathrm{mol} \mathrm{L}^{-1}$ acyclovir. The potential sweep rate was $50 \mathrm{mV} \mathrm{s}^{-1}$. (B) Typical cyclic voltammograms of $\mathrm{MCPE} / \mathrm{Nf}$ recorded in the absence (a) and presence (b) of $300 \mu \mathrm{mol} \mathrm{L}^{-1}$ acyclovir. The potential sweep rate was $50 \mathrm{mV} \mathrm{s}^{-1}$.

$$
I_{\mathrm{p}}=\left(n^{2} F^{2} / 4 R T\right) v A \Gamma^{*}
$$

where $I_{\mathrm{p}}$ is the peak current, $A$ the electrode surface area and $\Gamma^{*}$ the surface coverage of the redox species, $\Gamma^{*}$ can be obtained. Taking the average of both the cathodic and anodic currents, a total surface coverage of the electrode by the nickel species of about $4.55 \pm 0.13 \times 10^{-9} \mathrm{~mol} \mathrm{~cm}^{-2}$ was derived. In the higher range of the potential sweep rates of 150 to $1250 \mathrm{mV} \mathrm{s}^{-1}$ (Figs. 3D and 3F), this dependency is of the square-root form, signifying the dominance of the diffusion process as being the rate-limiting step in the total redox transition of the nanocomposite. This limiting-diffusion process, which has also been reported for some other Ni-based modified electrodes,,$^{10,23,24,28}$ may be due to charge neutralization during the oxidation/reduction process.

Study of the electrocatalytic oxidation of acyclovir on the MCPE/ Nf surface

Figure 4 shows cyclic voltammograms of CPE (A) and $\mathrm{MCPE} / \mathrm{Nf}$ (B) in the supporting electrolyte in both the absence (curve a) and presence (curve b) of $300 \mu \mathrm{mol} \mathrm{L}^{-1}$ acyclovir at a potential sweep rate of $50 \mathrm{mV} \mathrm{s}^{-1}$. Acyclovir represented a 


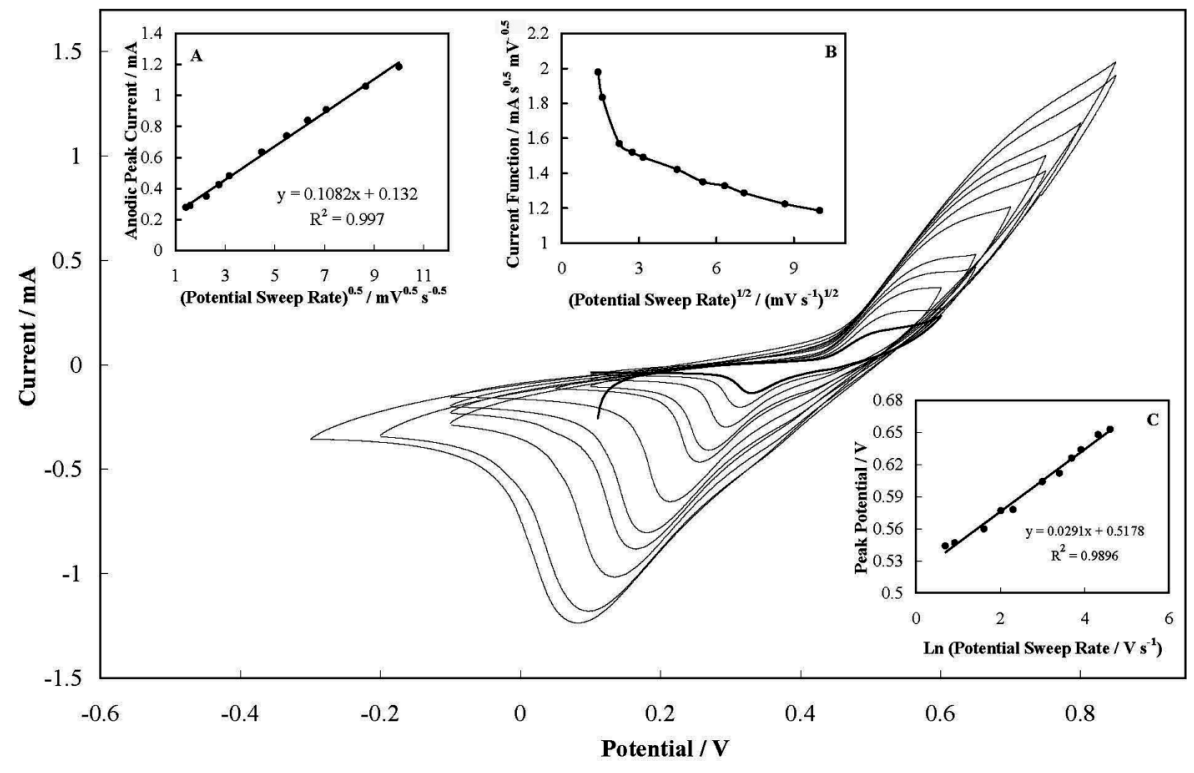

Fig. 5 Cyclic voltammograms recorded using MCPE/Nf in $100 \mathrm{mmol} \mathrm{L}^{-1}$ containing $300 \mu \mathrm{M}$ acyclovir and at different potential sweep rates. The potential sweep rate from the inner to the outer were: $2,2.5,5,7.5,10,20,30,40,50,75$ and $100 \mathrm{mV} \mathrm{s}^{-1}$. Inset: (A) Dependency of the anodic current to the square root of the potential sweep rate, (B) dependency of the current function to the square root of the potential sweep rate, (C) dependency of the anodic peak potential to the logarithm of the potential sweep rate.

weak anodic signal on CPE in the swept potential range, while it was oxidized on MCPE/Nf. The voltammograms represent an enhancement in the anodic peak current (and the corresponding charge) and decrements in the cathodic peak current (and the corresponding charge). From the cyclic voltammograms represented in Fig. 4, an electrocatalytic mechanism for the electrooxidation of acyclovir on the MCPE/Nf surface can be deduced in which the redox transition of the nickel species,

$$
\mathrm{Ni}(\mathrm{II}) \rightleftarrows \mathrm{Ni}(\mathrm{III})+\mathrm{e}^{-}
$$

was followed by the oxidation of acyclovir via the following reaction:

$$
\begin{aligned}
& \mathrm{Ni}(\mathrm{III})+\text { acyclovir } \stackrel{k_{\text {cat }}}{\longrightarrow} \text { intermediate/product( }(1)+\mathrm{Ni}(\mathrm{II}) \\
& \mathrm{Ni}(\mathrm{III})+\text { intermediate/product }(1) \stackrel{k_{\text {cat }}^{\prime}}{\longrightarrow} \operatorname{product}(2)+\mathrm{Ni}(\mathrm{II})
\end{aligned}
$$

Regarding the product(s) of the electrocatalytic oxidation of acyclovir, one electroreactive functional group of the drug can be oxidized in multiple steps and/or the simultaneous oxidation of electroreactive functional groups of the drug may occur. Acyclovir bears two major groups of the guanine analog and the first-type alcohol. As a guanine derivative, acyclovir can be oxidized to the corresponding oxoguanine analog: ${ }^{31,32}$<smiles></smiles><smiles></smiles>

In addition, as a primary alcohol, acyclovir, can also be oxidized to the corresponding aldehyde and/or carboxylic acid on nickel-based electrodes: ${ }^{33}$<smiles>Nc1nc2c(nc([PH2+])n2COCCO)c(=O)[nH]1</smiles><smiles>[14CH2]c1nc2c(=O)[nH]c(N)nc2[nH]1</smiles><smiles>[14CH2]c1nc2c(=O)[nH]c(N)nc2n1COCCO</smiles><smiles>[14CH2]c1nc2c(=O)[nH]c(N)nc2[nH]1</smiles>

Figure 5 shows cyclic voltammograms of MCPE/Nf in a range of potential sweep rates of 2 to $100 \mathrm{mV} \mathrm{s}^{-1}$ in the presence of $300 \mu \mathrm{mol} \mathrm{L}{ }^{-1}$ acyclovir. Upon increasing the potential sweep rate, the anodic peak current increased, and depended linearly on the square root of the potential sweep rate (Fig. 5, inset A). This linear dependency indicates the domination of a diffusion-controlling step for the electrooxidation process. Another point is that plotting the current function (peak current divided by the square root of the potential sweep rate) against the square root of the potential sweep rate revealed a negative slope (Fig. 5, inset B). This further confirms the electrocatalytic nature of the process. The value of the electron-transfer coefficient for the reaction can be obtained from the following equation: ${ }^{34}$

$$
E_{\mathrm{p}}=(R T / 2 \alpha F) \ln v+\text { constant }
$$

which is valid for a totally irreversible diffusion-controlled 


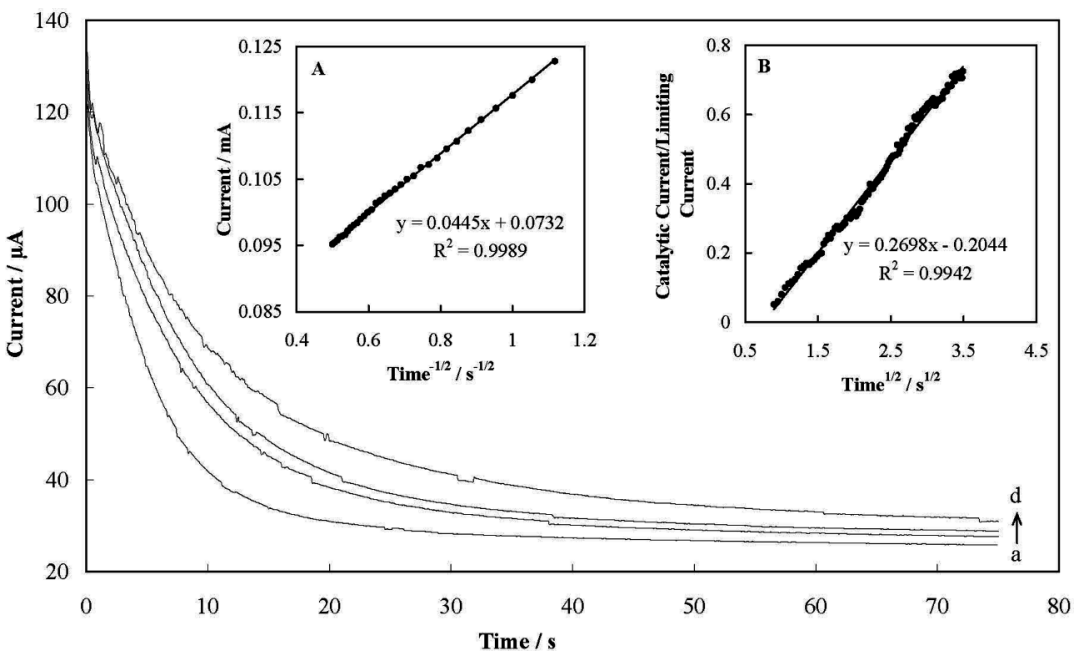

Fig. 6 Chronoamperograms recorded using MCPE/Nf in different concentrations of acyclovir of: (a) 0 , (b) 1.48 , (c) 2.91 , (d) $4.31 \mathrm{mM}$. Potential step was $600 \mathrm{mV}$. Inset: (A) Dependency of the transient current on $t^{-0.5}$ for curve c, (B) dependence of $I_{\text {cat }} / I_{\mathrm{L}}$ on $t^{0.5}$ for curve c.

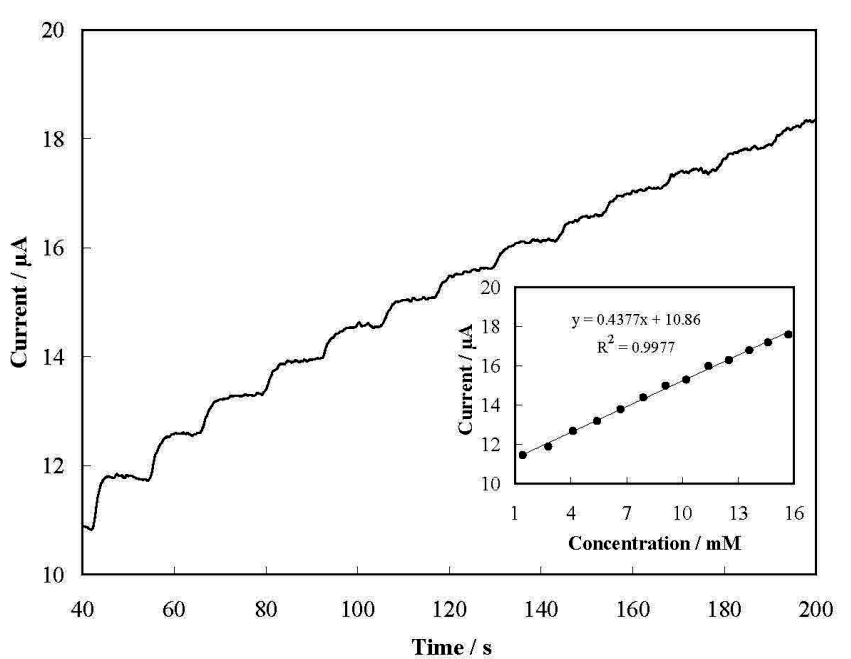

Fig. 7 Current signals as a function of time in PBS during repetitive injections of acyclovir in a batch system using ns-MCPE. The applied potential was $550 \mathrm{mV}$. Inset: Dependency of the transient current on the acyclovir concentration.

process. Using the dependency of the anodic peak potential on the natural logarithm of the potential sweep rate (Fig. 5, inset C), the value of the electron-transfer coefficient was obtained as $0.44 \pm 0.3$. This indicates a near-symmetric barrier for the potential energy change during the electrooxidation process with a slight curvature to the reaction products.

Figure 6 shows chronoamperograms recorded in the absence (curve a) and presence (curves b - d) of acyclovir using potential step of $600 \mathrm{mV}$. The transient currents decayed with time in a Cotrellian manner. This indicates that the electrocatalytic oxidation of acyclovir on MCPE/Nf was controlled by diffusion in the bulk of the solution. This was also confirmed by cyclic voltammetry (vide supra). By using the slope of the line represented in Fig. 6, inset A, the diffusion coefficient of acyclovir can be obtained according to Cottrell equation: ${ }^{30}$

$$
I=n F A D^{1 / 2} C \pi^{-1 / 2} t^{-1 / 2}
$$

Table 1 Determined parameters for the calibration curve of acyclovir and the accuracy and precision $(n=3)$ for the electrocatalytic oxidation of acyclovir on $\mathrm{MCPE} / \mathrm{Nf}$

$\begin{array}{lc}\text { Linear range/mM } & 1.4-15.7 \\ \text { Slope } / \mathrm{mA} \mathrm{M}^{-1} & 0.44 \pm 0.1 \\ \text { Intercept } / \mu \mathrm{A} & 10.86 \pm 0.2 \\ \mathrm{LOD} / \mathrm{mM} & 0.04 \\ \mathrm{LOQ} / \mathrm{mM} & 0.12 \\ \mathrm{RSD}, \%^{\mathrm{a}} & 3.13 \\ \mathrm{RSD}, \%^{\mathrm{b}} & 4.38 \\ \text { Bias, \% } & 1.17\end{array}$

a. Within day reproducibility.

b. Between day reproducibility.

c. The value was reported for the middle concentration of the calibration curve.

where $D$ is the diffusion coefficient, $A$ the electrode surface area and $C$ the bulk concentration. The mean value of the diffusion coefficient for acyclovir was found to be $4.66 \pm 0.06 \times$ $10^{-6} \mathrm{~cm}^{2} \mathrm{~s}^{-1}$.

Chronoamperometry can also be used to evaluate the catalytic rate constant according to the following equation: ${ }^{30}$

$$
I_{\text {cat }} / I_{\mathrm{L}}=\gamma^{1 / 2}\left[\pi^{1 / 2} \operatorname{erf}\left(\gamma^{1 / 2}\right)+\exp (-\gamma) / \gamma^{1 / 2}\right]
$$

where $I_{\text {cat }}$ and $I_{\mathrm{L}}$ are the currents in the presence and absence of acyclovir, respectively, and $\gamma=k_{\text {cat }} C t$ is the argument of the error function, $k_{\text {cat }}$ is the catalytic rate constant and $t$ is the elapsed time. In the cases where $\gamma>1.5, \operatorname{erf}\left(\gamma^{1 / 2}\right)$ is almost equal to unity, the above equation can be reduced to

$$
I_{\text {cat }} / I_{\mathrm{L}}=\gamma^{1 / 2} \pi^{1 / 2}=\pi^{1 / 2}\left(k_{\text {cat }} C t\right)^{1 / 2}
$$

From the slope of the $I_{\text {cat }} / I_{\mathrm{d}} v s . t^{1 / 2}$ plot, presented in Fig. 6, inset $\mathrm{B}$, the mean value of $k_{\mathrm{cat}}$ for the electrooxidation of acyclovir was obtained as $3.50 \pm 0.10 \times 10^{4} \mathrm{~cm}^{3} \mathrm{~mol}^{-1} \mathrm{~s}^{-1}$.

\section{Determination of acyclovir}

Typical amperometric signals obtained during successive increments of acyclovir to a $100 \mathrm{mmol} \mathrm{L}^{-1} \mathrm{NaOH}$ solution using 
Table 2 Determination and recovery of acyclovir in pharmaceutical formulations

\begin{tabular}{lcccccc}
\hline \multicolumn{1}{c}{ Sample type } & Amount labeled & Amount added & Amount found & Recovery, $\%$ & RSD, $\%$ & Bias, $\%$ \\
\hline Tablet-Sample 1 & $400 \mathrm{mg}$ & - & $383 \mathrm{mg}$ & 95.8 & 3.51 & 4.3 \\
Tablet-Sample 1 & - & $400 \mathrm{mg}$ & $388 \mathrm{mg}$ & 97.0 & 4.11 & 3.0 \\
Tablet-Sample 1 & - & $400 \mathrm{mg}$ & $387 \mathrm{mg}$ & 96.8 & 3.71 & 3.3 \\
Tablet-Sample 2 & $400 \mathrm{mg}$ & - & $391 \mathrm{mg}$ & 97.8 & 4.03 & 2.3 \\
Tablet-Sample 2 & - & $400 \mathrm{mg}$ & $385 \mathrm{mg}$ & 96.3 & 4.16 & 3.8 \\
Tablet-Sample 2 & - & $400 \mathrm{mg}$ & $387 \mathrm{mg}$ & 96.8 & 4.18 & 3.3 \\
Topical Cream-Sample 1 & $5 \%$ & - & $4.83 \%$ & 96.6 & 3.19 & 3.4 \\
Topical Cream-Sample 1 & - & $5 \%$ & $4.91 \%$ & 98.2 & 3.24 & 1.8 \\
Topical Cream-Sample 1 & - & - & $4.88 \%$ & 97.6 & 3.18 & 2.4 \\
Topical Cream-Sample 2 & $5 \%$ & $-5 \%$ & $4.81 \%$ & 96.2 & 3.42 & 3.8 \\
Topical Cream-Sample 2 & - & $5 \%$ & $4.87 \%$ & 96.4 & 4.11 & 3.6 \\
Topical Cream-Sample 2 & - & & & 97.4 & 3.19 & 2.6 \\
\hline
\end{tabular}

MCPE/Nf are depicted in Fig. 7. Gentle stirring for a few seconds was needed to promote solution homogenization after each injection. The electrode response is quite rapid, and proportional to the acyclovir concentration. The corresponding calibration curve for the amperometric signals is shown in the inset of Fig. 7. The limits of detection (LOD) and quantitation (LOQ) of the procedure were calculated according to the $3 \mathrm{SD} / m$ and $10 \mathrm{SD} / m$ criteria, respectively, where $\mathrm{SD}$ is the standard deviation of the intercept, and $m$ is the slope of the calibration curves. ${ }^{35}$ The determined parameters for calibration curves of the drug are reported in Table 1 .

The applicability of the proposed amperometric method for the sample dosage form was examined by analyzing acyclovir tablets and topical creams. It was found that the amounts of drug, determined through this method, are in good agreement with the reported values (Table 2).

In order to evaluate the accuracy of this method and to find out whether the excipients in pharmaceutical dosage form show any interference with the analysis, the proposed amperometric method was checked by recovery experiments using the standard addition method (Table 2). The results indicate the absence of interference from commonly encountered pharmaceutical excipients used in the selected formulations.

In order to verify the durability and long-term stability of the MCPE/Nf, 50 consecutive cyclic voltammograms using this electrode were recorded in the supporting electrolyte. It was found that the peak currents changed slightly $(<3 \%)$. In addition, the electrode was stored in the supporting electrolyte when not in use, and retained its electroreactivity for 2 weeks.

In order to investigate the fouling resistance of MCPE/Nf, consecutive cyclic voltammograms using MCPE/Nf in a $300 \mu \mathrm{mol} \mathrm{L} \mathrm{L}^{-1}$ acyclovir solution were recorded. It was observed that the current as well as the potential of the peak related to the acyclovir electrooxidation changed slightly $(<4 \%)$ after 50 cyclic scans.

The selectivity of the amperometric method for the assay of the drug was examined in the presence of some common interferences (glucose, L-tyrosine, ascorbic acid, L-serine, sucrose, dopamine and uric acid) and some common excipients usually used in pharmaceutical preparations (microcrystalline cellulose, colloidal silicon dioxide, titanium dioxide, talc, starch, magnesium stearate, liquid paraffin, white soft paraffin, cetostearyl alcohol and poloxyl 20 cetostearyl ether). The results showed that under the experimental conditions applied in this work, no significant interference was observed for these compounds due to insolubility in the electrolyte, or rejection of the diffusion of the negatively charged compounds by the Nafion membrane.

\section{Conclusions}

Nanoporous nickel microspheres were prepared with hydrazine hydrate as a reducing agent in a Triton X-100/cyclohexane/water nanoemulsion. The microspheres were successfully applied to modification of a carbon paste electrode. The modified electrode represented high catalytic activity toward electrooxidation of the drug acyclovir via a cyclic mediation electron-transfer process. The electrocatalytic activity of the microspheres was higher than that of the microstructured nickel species, due to the high surface area, ease of electrolyte penetration into the nanopores and/or the nanosize effect of the microspheres. An amperometric procedure was successfully developed for the quantification of acyclovir with high sensitivity in bulk and pharmaceutical formulations. The microspheres can have potential applications in other areas of electroanalysis and energy storage systems.

\section{Acknowledgements}

We would like to thank the Research Councils of Islamic Azad University and Shiraz University of Medical Sciences (90-01-36-4208) for supporting this research.

\section{References}

1. P. Zijlstra and M. Orrit, Rep. Prog. Phys., 2011, 74, Article Number: 106401.

2. B. M. Munoz-Flores, B. I. Kharisov, V. M. Jimenez-Perez, P. E. Martinez, and S. T. Lopez, Ind. Eng. Chem. Res., 2011, 50, 7705 .

3. S. J. Guo and E. K. Wang, Nano Today, 2011, 6, 240.

4. G. Thirumurugan and M. D. Dhanaraju, Adv. Sci. Lett., 2011, 4, 339.

5. E. Comini and G. Sberveglieri, Mater. Today, 2010, 13, 28.

6. A. Kolmakov and M. Moskovits, Annu. Rev. Mater. Res., 2004, 34, 151.

7. N. Sattarahmady, H. Heli, and F. Faramarzi, Talanta, 2010, 82, 1126.

8. K. R. Prasad and N. Miura, Appl. Phys. Lett., 2004, 85, 4199.

9. L. D. Rafailovic and D. M. Minic, Hemijska Industrija, 2009, 63, 557.

10. N. Sattarahmady, H. Heli, and A. A. Moosavi-Movahedi, Biosens. Bioelectron., 2010, 25, 2329.

11. Z. W. Shi and A. V. Walker, Langmuir, 2011, 27, 11292. 
12. N. Chopra, W. W. Shi, and A. Bansal, Carbon, 2011, 49, 3645.

13. M. Cabo, S. Garroni, E. Pellicer, C. Milanese, A. Girella, A. Marini, E. Rossinyol, S. Surinach, and M. D. Baro, Int. J. Hydrogen Energy, 2011, 36, 5400.

14. T. R. Ling, C. S. Li, J. J. Jow, and J. F. Lee, Electrochim. Acta, 2011, 56, 1043.

15. J. H. Li, C. C. Wang, C. J. Huang, Y. F. Sun, W. Z. Weng, and H. L. Wan, Appl. Catal., A, 2010, 382, 99.

16. S. K. Meher, P. Justin, and G. R. Rao, Electrochim. Acta, 2010, 55, 8388 .

17. D. D. Zhao, W. J. Zhou, and H. L. Li, Chem. Mater., 2007, 19, 3882.

18. J. M. Gutiérrez, C. Gonzalez, A. Maestro, I. Sole, C. M. Pey, and J. Nolla, Curr. Opin. Colloid Interface Sci., 2008, 13, 245.

19. I. Capek, Adv. Colloid Interface Sci., 2004, 110, 49.

20. Z. Jia, W. Yujun, and L. Guangsheng, React. Funct. Polym., 2005, 65, 249.

21. A. K. Ganguli, A. Ganguly, and S. Vaidya, Chem. Soc. Rev., 2010, 39, 474.

22. I. Svancara, A. Walcarius, K. Kalcher, and K. Vytras, Central Eur. J. Chem., 2009, 7, 598.

23. H. Heli, A. Jabbari, S. Majdi, M. Mahjoub, A. A. Moosavi-Movahedi, and Sh. Sheibani, J. Solid State Electrochem., 2009, 13, 1951.
24. S. Majdi, A. Jabbari, H. Heli, and A. A. Moosavi-Movahedi, Electrochim. Acta, 2007, 52, 4622.

25. Y. Mu, D. Jia, Y. He, Y. Miao, and H.-L. Wu, Biosens. Bioelectron., 2011, 26, 2948.

26. E. Laviron, J. Electroanal. Chem., 1979, 101, 19.

27. H. Luo, Z. Shi, N. Li, Z. Gu, and Q. Zhuang, Anal. Chem., 2001, 73, 915.

28. M. Hajjizadeh, A. Jabbari, H. Heli, A. A. Moosavi-Movahedi, and S. Haghgoo, Electrochim. Acta, 2007, 53, 1766.

29. S. Berchmans, H. Gomathi, and G. P. Rao, J. Electroanal. Chem., 1995, 394, 267.

30. A. J. Bard and L. R. Faulkner, "Electrochemical Methods: Fundamentals and Applications", 1980, John Wiley and Sons, New York.

31. K. C. Honeychurch, M. R. O'Donovan, and J. P. Hart, Biosens. Bioelectron., 2007, 22, 2057.

32. E. Gonzalez-Fernandez, N. de-los Santos-Alvarez, M. J. Lobo-Castanon, A. J. Miranda-Ordieres, and P. TunonBlanco, Electroanalysis, 2008, 20, 833.

33. O. Hammerich, J. H. P. Utley, and L. Eberson (ed.), "Organic Electrochemistry", 1991, Marcel Dekker, New York.

34. J. A. Harrison and Z. A. Khan, J. Electroanal. Chem., 1970, $28,131$.

35. J. C. Miller and J. N. Miller, "Statistics for Analytical Chemistry", 4th ed., 1994, Ellis-Horwood, New York. 